\title{
Cambio de titularidad empresarial. Garantía a favor y no frente a los trabajadores. Supuestos de crisis empresarial y nueva titularidad de Cooperativas o Sociedades Anónimas Laborales
}

TOMÁS IGLESIAS PÉREZ *

\begin{abstract}
SUMARIO: I. La regulación de la sucesión, garantía de los trabajadores. II. Una excepción en la interpretación de la sucesión empresarial; los supuestos en que el nuevo titular es una cooperativa o sociedad anónima laboral. III. La excepción de la excepción, que confirma la regla.
\end{abstract}

I. La regulación de la sucesión. Garantía de los trabajadores

Es un tema pacífico que la regulación legal de la sucesión de empresa contenida en el art. 44 del Estatuto de los Trabajadores no es sino una garantía de éstos al establecerse que cuando se produce un cambio de titularidad empresarial no se extingue el contrato de trabajo sino que el nuevo empresario queda subrogado en los derechos y obligaciones laborales del anterior.

A favor de la naturaleza garantista de esta regulación está la propia literalidad de la legislación, los pronunciamientos de la jurisprudencia y las posiciones doctrinales. Respecto a la legislación, basta llamar la atención acerca de que el mencionado art. 44 del Estatuto de los Trabajadores se ubica en la sección $2^{\mathrm{a}}$ del capítulo III de dicha norma legal, siendo la rúbrica de dicha sección precisamente la de: "Garantías por cambio del empresario". Tampoco puede olvidarse que la Directiva Comunitaria 77/187, de 14-2-77, existente en la materia, se refiere precisamente a «la aproximación de las legislaciones de los Estados miembros relativas al mantenimiento de los derechos de los

\footnotetext{
* Abogado.
} 
trabajadores en caso de traspasos de empresas, de centros de actividad o de partes de centros de actividad".

Respecto a los pronunciamientos de la jurisprudencia vamos a limitarnos a señalar los efectuados en dos ocasiones por el Tribunal Supremo que resultan clarificadores y contundentes al respecto; nos referimos a la Sentencia del Tribunal Supremo de 16-6-83 (R.A. 3017) cuando refiriéndose a la subrogación empresarial indica que: "constituye una firme garantía de la estabilidad del puesto de trabajo y con tal criterio teleológico como primario y fundamental ha sido constante y uniformemente interpretado por la doctrina legal de esta Sala"; del mismo Tribunal puede citarse la Sentencia de 12-7-88 (R.A. 5809), al valorar el art. 44 del E.T. como "una nueva expresión legislativa de la voluntad del sistema de proteger la estabilidad en el empleo, por cuanto inequivocamente no se encuentra situado en el ámbito de otra persona y sí de una empresa, instituto jurídico con vocación de permanencian; a su vez, esta resolución hace mención a las Sentencias del Tribunal de Justicia de las Comunidades Europeas en varias cuestiones prejudiciales sobre interpretación de la Directiva 77/187 del Consejo, antes citada, entre las que destacan las Sentencias e 18 de Marzo de 1986 y de 17 de Diciembre de 1987.

También en la doctrina MARTIN VALVERDE se ha referido a que cel fundamento de esta regulación es claro... La Ley opta por la estabilidad del empleo de los trabajadores ocupados en la empresa cuya titularidad se ha transmitido..."1. Pero ha sido seguramente GONZALEZ BIEDMA uno de los autores que se ha referido al tema incorporando matices de más interés; refiriéndose a que tradicionalmente se ha estimado que mediante la normativa subrogatoria se tratan de proteger tres tipos de intereses: el del empresario, el de la economía general y el de los trabajadores, concluye afirmando que: «sin apenas excepciones, tanto la jurisprudencia como la doctrina han venido a coincidir en afirmar que era sin duda el interés del trabajador el primordialmente tutelado...n; y en la argumentación desarrollada en apoyo de tal afirmación insiste, además de en el dato anteriormente expuesto del emplazamiento sistemático del art. 44 del E.T., en dos razones: en primer lugar, se refiere el autor a que "la propia sede en la que se halla inserta la normativa subrogatoria,... es la de un texto normativo de carácter laboraln, pareciendo previsible que si el interés preponderantemente protegido hubiera sido cualesquiera de los otros citados la regulación habría estado contenida en normas civiles o mercantiles; en segundo lugar, se refiere a "la evidencia de que si hay alguien objetivamente beneficiado por la normativa... es el trabajador ${ }^{2}$.

1 MARTIN VALVERDE, A; RODRIGUEZ SAÑUDO, F. y GARCIA MURCIA, J.: Derecho del Trabajo, $3^{n}$ edición, pág. 576.

2 GONZALEZ BIEDMA, E.: El cambio de titularidad de la empresa en el Derecho del Trabajo, págs. 38 a 47. 


\section{Una excepción en la interpretación de la sucesión empresarial: los supuestos en que el nuevo titular es una cooperativa o sociedad anónima laboral}

Desde hace ya algunos años ha venido emergiendo a través de las resoluciones de los Tribunales de Justicia una doctrina novedosa en el materia que nos ocupa. La existencia de crisis empresariales que se han saldado con la extinción de los contratos de trabajo, pero que han sido seguidas del intento de los trabajadores desempleados de iniciar un nuevo proyecto en forma de Cooperativa o S.A.L. que les garantizase su empleo, ha dado lugar a una particular consideración del cambio de titularidad en estos supuestos, concluyéndose que no se da sucesión empresarial ni subrogación al no ser de aplicación el art. 44 del Estatuto de los Trabajadores. Un primer dato, ya indicado, conviene destacar: que emerge, se consolida y aplica esta doctrina a través de la elaboración jurisprudencial. Junto a ello y sobre los motivos de fondo que le sirven de justificación conviene destacar un aspecto clave al que se refirí de forma contundente una Sentencia del Tribunal Superior de Justicia de Cataluña: "no puede hacerse aplicación de un precepto establecido en garantía de los trabajadores frente a los trabajadores mismos» 3 .

Los pronunciamientos que se han venido efectuando respecto a la cuestión que no ocupa se han podido producir habitualmente en tres clases de litigios:

- en pleitos en los cuales algunos trabajadores "descolgados" de la iniciativa colectiva protagonizada por el conjunto de sus compañeros, reclamasen a la Cooperativa o S.A.L. en que éstos estuviesen asociados o bien indemnizaciones por despidos del antiguo titular, o bien cantidades adeudadas por éste...;

- en procedimientos donde el Fondo de Garantía Salarial se hubiese negado a hacer frente a indemnizaciones por las extinciones contractuales producidas por el antiguo empresario, arguyendo que no había tal ruptura del nexo contractual al existir sucesión de empresa y subrogación de la Cooperativa o S.A.L. en los derechos y obligaciones del anterior titular; $y$

- en contenciosos donde se hubiese discutido la procedencia de que la Tesorería General de la Seguridad Social se hubiera dirigido contra las nuevas personas jurídicas integradas por los trabajadores, tra-

${ }^{3}$ S.T.S.J. de Cataluña de 11-2-92 (R.A. 806) F.J. $2^{\text {a }}$ 
tando de recuperar las cotizaciones adeudadas por la empresa que había sido víctima de la crisis.

Teniendo en cuenta lo anterior tiene interés subrayar que la contestación a si existe o no sucesión de empresa y subrogación ha de ser unitaria para todos los supuestos. En cada caso hay que llegar a la conclusión de que o existe o no existe sucesión; no cabe pensar que en un determinado supuesto hay sucesión de empresa respecto a las reclamaciones de los trabajadores no integrados en la S.A.L. o Cooperativa debiendo accederse a sus pretensiones, y por ejemplo no exista en el mismo caso tal sucesión en relación a las indemnizaciones que correspondiera pagar al Fondo de Garantía Salarial, debiendo dicho organismo hacer frente a las mismas; tampoco cabe pensar que el FOGASA tuviera que responder de las indemnizaciones porque no hubo sucesión, y en el mismo caso la T.G.S.S. sí pudiera hacer reclamación de las cuotas pendientes entendiendo que hubo sucesión empresarial.

En definitiva, si existe sucesión tienen derecho a reclamar tanto los trabajadores del antiguo empleador como la TGSS por las cotizaciones adeudadas por áquel y, por supuesto, nada tiene que pagar el FOGASA por la extinción del contrato con el primitivo empresario porque no existe tal ruptura. Pero si no hay sucesión no tiene que responder este último organismo por las indemnizaciones y nada tienen que reclamar ni la TGSS ni los antiguos trabajadores.

Para recalcar la necesidad de una respuesta uniforme, para todas las vertientes y aspectos, a la interrogante de si existe o no sucesión convierne que nos detengamos en primer lugar en el aspecto antes aludido de las cotizaciones de Seguridad Social. El art. 104,1 del Texto Refundido de la Ley General de la Seguridad Social, aprobada por el Real Decreto Legislativo 1/94, de 20 de Junio, se refiere a la responsabilidad empresarial de cumplimiento de la obligación de cotización e indica que responderán, entre otras, la persona señalada en el apartado 2 del art. 127 de la misma Ley, a saber, el adquirente en los casos de sucesión. Pero debe quedar claro que esta regulación no es autónoma ni protege intereses distintos a los del art. 44 del E.T. sino que no es sino desarrollo, reflejo y consecuencia de éste. Buena prueba de ello es que incluso cuando el citado art. 104,1 regula la obligación empresarial de cotizar y se remite precisamente al art. 127,2 para determinar otras personas igualmente responsables, lo hace a un precepto como éste, donde se regula cómo el adquirente de una empresa responderá solidariamente con el anterior de las prestaciones de Seguridad Social a que éste tuviera que hacer frente y hubieran sido causadas con anterioridad a la sucesion. Con ello se vuelve a poner el énfasis en que, determinada la existencia de sucesión, el nuevo empleador pecha con sus responsabilidades laborales respecto a los 
trabajadores, las que pudieran corresponderle en materia de prestaciones de Seguridad Social y también las correspondientes a las cuotas adeudadas a la TGSS; pero, al contrario, si no hay sucesión ni subrogación, no lo existe para ninguna de tales obligaciones.

Conviene ahora que volvamos a la fundamentación que ha podido servir de base a la excepción que en los supuestos que contemplamos ha venido a configurarse a la hora de interpretar la sucesión y subrogación empresarial. Si anteriormente, hicimos mención a que el argumento "clave" de toda la elaboración se centra en que «no puede hacerse aplicación de un precepto establecido en garantía de los trabajadores frente a los trabajadores mismos", no es menos cierto que al pronunciarse sobre los distintos casos analizados los Tribunales de Justicia han ido elaborando algunos otros argumentos centrales que sirven de desarrollo y de ampliación a áquel, los que pasamos a exponer.

$1^{\circ}$. Una primera línea de razonamiento insiste en la idea de que no parece razonable cargar con las responsabilidades del antiguo titular a quienes esforzándose para mantener sus puestos de trabajo han invertido en la nueva iniciativa las cantidades percibidas tanto por las prestaciones de desempleo, como por las indemnizaciones debidas a causa de la extinción de sus contratos de trabajo, con mucha frecuencia procedentes del Fondo de Garantía Salarial dada la crisis empresarial precedente; en ocasiones también se añade que se arriesgan incluso los créditos personalmente contraidos para poner en marcha la nueva empresa ${ }^{4}$.

$2^{\circ}$. Un segundo argumento se utiliza en las resoluciones judiciales en apoyo de la interpretación que venimos glosando, y que si guarda alguna relación con el anterior incorpora un matiz diferente que lo completa. Así se hace reiterada mención a que, privando a los trabajadores de las prestaciones de desempleo que son consecuencia de la extinción del contrato con el antiguo empleador, así como de las indeminizaciones que no son sino efecto de dicha ruptura y, por el contrario, cargándoles con las responsabilidades y obligaciones de hacer frente a las que incumplió el antiguo empresario respecto a los trabajadores que no se hayan sumado al nuevo proyecto, o en relación a las cotizaciones debidas a la TGSS se estaría poniendo en peligro $y$ riesgo la estabilidad y aun la propia existencia de la nueva iniciativa ${ }^{5}$

1 Sentencias del Tribunal Supremo de 20-7-88 (R.A. 6211) y del T.S.J. de Cataluña de 2811-91 (R.A. 6511) y de 4-12-93 (Actualidad Laboral $n^{\circ} 26$, referencia 867).

5 Sentencias del Tribunal Supremo de 20-7-88, ya citada, y del T.S.J. de Cataluña de 1-1091 (R.A. 5613). 
$3^{\circ}$. Una tercera reflexión se ha ido consolidando para dar consistencia global a la interpretación a que nos venimos refiriendo; se insiste en un elemento que pone de relieve y subraya la ruptura entre la antigua y la nueva empresa hasta el punto de que evita que pueda considerarse que hay sucesión entre una y otra: la diferencia en cuanto a los planteamientos y filosofía de los dos fenómenos empresariales y asociativos, que se valoran como de tal calado que resulta incompatible con la existencia de sucesión empresarial; las sentencias que a este respecto se pronuncian concretan los mencionados planteamientos y filosofías refiriéndose a determinados aspectos sobre la constitución, los fines, la planificación, el sistema de funcionamiento y el enfoque empresarial en unos y otros casos.

$4^{\circ}$. Una cuarta idea completa, junto a los anteriores, la interpretación a que nos referimos. Partiendo de que uno de los requisitos fácticos sobre los cuales se elaboran estos pronunciamientos es la existencia previa de una crisis importante de la antigua empresa, normalmente los nuevos titulares no van a beneficiarse de la transmisión de elementos inmateriales que resultan sumamente importantes en el desenvolvimiento empresarial cuales son la cartera de clientes, las líneas crediticias, la propia imagen empresarial...; estos elementos en ningún caso van a ser recibidos por la nueva entidad que se trata de desarrollar de forma similar a lo que ocurre en un cambio de titularidad típico, sino que por el contrario o no serán recibidos por la Cooperativa o S.A.L., o serán recuperados después de arduos esfuerzos y por el transcurso del tiempo que vaya afianzando ante proveedores, clientes, y entidades financieras la viabilidad y fortaleza del nuevo proyecto ${ }^{7}$.

Entiendo que son las cuatro líneas argumentales expuestas las que, recogidas con más o menos énfasis unas u otras en las distintas resoluciones judiciales, han ido dando consistencia a la nueva interpretación del art. 44 del E.T. en los supuestos contemplados. A todas ellas les une esa orientación que antes se apuntó de defensa del principio de estabilidad en el empleo y de que una institución configurada como garantía de los trabajadores no terminase operando contra los intereses de los mismos. Ahora bien, si esas líneas las podemos deducir de la contemplación de un conjunto amplio de pronunciamientos, no conviene olvidar que al sentido del fallo o a la orientación de éstos se flega a través de una visión particularizada en cada supuesto. Porque si para apreciar en general si existe o no un supuesto de sucesión empresarial con el alcance del art. 44 del E.T., y comportando las obligaciones que de

7 Sentencia del T.S.J. del País Vasco de 28-9-93 (R.A. 4138). 
ello se derivan, se hace preciso ese análisis concreto de cada situación, no ocurre algo distinto para nuestro supuesto ${ }^{8}$. Es fácil pensar en, al menos, tres razones para que ello sea así: en primer lugar, que, como se ha indicado, si para cualquier supuesto de sucesión que implique la determinación de importantes responsabilidades para el nuevo titular así se exige, no existe ninguna causa para que no ocurra lo mismo en las ocasiones a que venimos refiriéndonos; en segundo lugar, porque habrá que aquilatar en ocasiones con extremo cuidado, pues no es el mismo caso el de una Cooperativa o S.A.L. protagonizada por la mayoría del colectivo de trabajadores afectados, al que no han querido sumarse algunos de ellos y que quisieran posteriormente dirigirse contra la nueva entidad de sus compañeros, que otro supuesto posible en que unos pocos trabajadores continuasen con la actividad empresarial precedente $\mathrm{y}$, al menos parcialmente beneficiándose de ella, marginando a la mayoría del colectivo afectado; en tercer lugar, por las posibles situaciones de fraude de ley que al socaire de la doctrina que comentamos pudieran llevarse a cabo, pero a ello nos referiremos en el apartado siguiente.

\section{La excepción de la excepción, que confirma la regla}

Si hacemos un análisis detenido de los pronunciamientos judiciales sobre el tema que nos ocupa, veremos que en ocasiones a pesar de existir crisis empresarial y nueva actividad posterior a ésta protagonizada por una Cooperativa o S.A.L. integrada por trabajadores vinculados en su día al primitivo empresario, sin embargo, se llega a la conclusión, contra lo que vienen siendo las referencias anteriores, de que sí existe sucesión de empresa. Nos interesa analizar las razones de ello para entender su alcance y sentido.

$Y$ a este respecto, tiene particular interés que nos centremos en algunos pronunciamientos del Tribunal Supremo recaídos en Recursos de Casación para la Unificación de Doctrina. Pero antes de entrar en los mismos conviene señalar que la sentencia decisiva de este Tribunal para marcar la orientación general a que dedicamos nuestras reflexiones es la de 20-7-88, citada anteriormente; a ella con reiteración se remiten las sentencias de los Tribunales Superiores de Justicia y las posteriores a dicha fecha de la Sala de lo Social del Tribunal Supremo.

Un primer supuesto significativo tiene lugar en una Sentencia de dicho Tribunal ${ }^{9}$ recaída en un recurso que impugnaba otra del T.S.J. de Cataluña,

B Sentencia del Tribunal Supremo de 20-3-93 (R.A. 1874).

9 Sentencia del T.S. de 20-3-93, ya citada. 
que había estimado el criterio del FOGASA acerca de que en un caso concreto de los que estudiamos sí había sucesión; un primer dato que llama la atención se refiere a que la parte recurrente alegaba como sentencias contradictorias con las que impugnaba 26 sentencias de la misma Sala (T.S.J. de Cataluña), lo que es expresivo de la frecuencia de los pronunciamientos al respecto y del grado de consolidación de la doctrina referida; otro aspecto que se observa en la resolución del T.S. se refiere a que si en el caso concreto contemplado la Sala había producido un cambio de criterio "ésta lo razona suficientemente»; y, en último lugar, interesa destacar cómo en el Fundamento de Derecho $4^{\circ}$ de la sentencia que venimos comentando se sintetizan tales razones que justifican el cambio de criterio en el supuesto concreto, haciéndose referencia como elementos fácticos determinantes, entre otros, a los siguientes:

- cuando se constituye la S.A.L. participan como socios 31 de los trabajadores afectados por el expediente de regulación de empleo, además del Administrador Gerente de la misma sociedad (empleadora anterior) y suscriben cada uno 26 acciones salvo el Gerente que suscribio 226 acciones, siendo éste último nombrado Presidente del Consejo de Administración y Consejero Delegado de la S.A.L.; y

- la adquisición del patrimonio único de la antigua empresa se efectúa mediante "entrega" a los trabajadores en virtud de una conciliación administrativa en la que interviene en nombre de la S.A. que era la antigua empleadora, quien más tarde fue nombrado Consejero Delegado de la S.A.L.

El segundo pronunciamiento a que me quería referir se produce en otra Sentencia del Tribunal Supremo ${ }^{10}$ igualmente recaída en un Recurso de la misma naturaleza que en el caso anterior, y argumentándose la excepcionalidad al criterio general aplicado en estos supuestos precisamente porque ise constituyó por tres de los trabajadores, familiares directos del anterior empresario, la S.A.L... detentando la propiedad de las acciones dichos hijos y familiares directos del primitivo empresario individual, además de otros parientes, que pasaron a ostentar con caracter único los cargos directivos de la nueva S.A.L.».

En definitiva, lo que vienen a poner de relieve los anteriores pronunciamientos, es que sí existe sucesión y subrogación empresarial, pese a la existencia de una crisis y la constitución de una Cooperativa o S.A.L., siempre que exista en dicho tránsito connivencia entre el antiguo empresario y los tra-

10 Sentęncia del T.S. de 22-12-93 (R.A. 9984). 
bajadores integrados en tales entidades; siempre que se trate de una argucia mediante la que el antiguo empleador siga ostentando por sí o por persona interpuesta el control y la dirección de la nueva entidad; cuando, en fin, no exista sino una actuación en fraude de ley.

Existe una regla que garantiza el art. 44 del E.T. con carácter general; un tratamiento excepcional, velando precisamente por la protección de la estabilidad en el empleo de los trabajadores en el supuesto típico a que hemos dedicado el presente trabajo; y a su vez una excepción a este tratamiento excepcional, cuando al socaire del mismo trata de introducirse una ficción, no habiendo en realidad ruptura auténtica entre los intereses del anterior empleador y de los trabajadores que asociados se esfuerzan por mantener su puesto de trabajo; pero esta excepción al tratamiento excepcional no hace sino confirmar la regla que éste ha venido configurando y las sólidas razones que le sirven de respaldo. 